1 O. Oldenberg, J. Opt. Soc. Amer. 61, 1092, 1098 [1971].

2 H. H. Brömer and F. SPIEweck, Z. Phys. 184, 481 [1965].

3 B. Brocklehurst and R. M. Duckworth, J. Phys. B 1, 990 [1968].

4 H. H. Brömer and F. Döbler, Z. Phys. 185, 278 [1965].

5 H. H. Brömer and J. Hesse, Z. Phys. 219, 269 [1969].

6 A. R. DeMonchy, Thesis, University of Amsterdam 1970.

7 I. R. Hurle, J. Chem. Phys. 41, 3592 [1964].

8 B. Brocklehurst and F. A. Downing, J. Chem. Phys. 46, 2976 [1967].
9 A. M. BAss, J. Chem. Phys. 40, 695 [1964]. - Y. TANAKA, F. R. Innes, A. S. Jursa, and M. Nakamura, J. Chem. Phys. 42, 1183 [1965].

10 S.-L. Chen and J. M. Goodings, J. Chem. Phys. 50, 4335 [1969].

11 J. A. Meyer, D. W. Setser, and W. G. Clark, J. Phys. Chem. 76, 1 [1972].

12 A. R. Lee and N. P. Carleton, Phys. Lett. 27 A, 195 [1968].

13 B. Brocklehurst, Nature Phys. Sci. 236, 12 [1972] and references therein.

14 J. Anketell and B. Brocklehurst, unpublished work.

\section{Low-temperature Heat Capacity and Paramagnetic Susceptibility Measurements on some Tetragonal Nickel(II) Compounds}

\author{
F. W. KLAAIJSEN \\ Kamerlingh Onnes Laboratory, State University, Leiden \\ The Netherlands \\ J. ReedijK * and H. T. Witteveen \\ Gorlaeus Laboratory, State University, Leiden \\ The Netherlands \\ (Z. Naturforsch. 27 a, 1532-1534 [1972] ; received 8 September 1972)
}

Zero-field splittings have been determined for the spin triplet ground state of $\mathrm{Ni}$ (II) in $\mathrm{Ni}$ (pyrazole) ${ }_{4} \mathrm{Cl}_{2}$ and $\mathrm{Ni}$ (pyrazole) ${ }_{4} \mathrm{Br}_{2}$, by means of heat capacity measurements in the $1-80 \mathrm{~K}$ region and by paramagnetic susceptibility measurements in the $2-80 \mathrm{~K}$ region.

The results of both measurements can be fitted with theory using the spin Hamiltonian

$$
H=g \beta H S+D\left[S_{z}^{2}-S(S+1) / 3\right]+E\left(S_{x^{2}}-S_{y}{ }^{2}\right)
$$

with the parameter $D$ equal to $7.2 \mathrm{~cm}^{-1}$ for the chloride and $5.4 \mathrm{~cm}^{-1}$ for the bromide. The value of $E$ appeared to be close to zero for both compounds, indicating nearly axial symmetry.

The anisotropic $g$-values (calculated from the susceptibility) are $g_{\perp}=2.21 \pm 0.03$ and $g_{\|}=2.14$ for the chloride and $g_{\perp}=2.20, g_{\| \mid}=2.12$ for the bromide.

\section{Introduction}

Nickel(II) ions $\left(3 \mathrm{~d}^{8}\right)$ with axially distorted octahedral coordination are known to yield a spin splitting of the ground state $\left({ }^{3} A_{2}\right)$, even in the absence of a magnetic field ${ }^{1}$. Usually these splittings are of the order of $0.1-1.0 \mathrm{~cm}^{-1}$ and are caused by second-order pin-orbit coupling. The magnitude of this splitting, $D$, deends upon the orbital splittings in the excited states (1. c. ${ }^{1,2}$ ).

Liehr and BALlHausen ${ }^{3}$ derived $D$ from the orbital splittings by means of the relation $D=-9 k \lambda^{2} / \Delta^{2}$, with $k=$ the magnitude of the trigonal ${ }^{3} \mathrm{~T}_{2} \mathrm{~g}$ splitting, $\lambda=$ spin-orbit coupling constant and $\Delta=$ distance between

* Reprint requests to Dr. J. REEDiJK, Department of Chemistry, State University, P.O. Box 75, Leyden, The Netherlands. the ${ }^{3} \mathrm{~A}_{2 g}$ and ${ }^{3} \mathrm{~T}_{2 g}$ term. In a previous study ${ }^{4-6}$ on the spectra, structure and bonding of tetragonal pyrazole compounds, we found unusually large orbital splittings for a number of compounds $\mathrm{Ni}$ (ligand $)_{4}$ (anion) $)_{2}$. This would yield zero-field splitting much larger than $1 \mathrm{~cm}^{-1}$, using the relation of LIEHR and BALLHAUSEN ${ }^{3}$.

In order to examine if a relation exists between $D$, the orbital splitting and the nature of the ligand and the anion, we determined $D$-values for a number of compounds of formula $\mathrm{NiL}_{4} \mathrm{X}_{2}$, in which $\mathrm{L}$ is a pyrazole or imidazole ligand, and $\mathrm{X}$ is a halide or nitrate anion. The $D$-values were determined with the aid of specific heat and paramagnetic susceptibility measurements. The results for two typical examples are discussed in the present paper.

\section{Experimental}

$\mathrm{Ni}$ (pyrazole) ${ }_{4} \mathrm{Cl}_{2}$ and $\mathrm{Ni}$ (pyrazole) ${ }_{4} \mathrm{Br}_{2}$ were prepared from the metal salt hydrates by adding the ligand in the ratio $1: 4$ in ethanol as a solvent. The compounds were checked for purity by chemical analysis. The crystal structures of both compounds have been published $^{7,8}$.

The heat capacity measurements were performed with the usual heat pulse method. To cool the sample to bath temperature a mechanical heat switch was used. The powdered salts were pressed into a thin walled gold-plated finned copper calorimeter to improve internal heat contact ${ }^{\boldsymbol{9}}$.

The paramagnetic susceptibility was measured by means of a PAR vibrating sample magnetometer, model 150 , in the $2-80 \mathrm{~K}$ region at fields around 5000 Oersted.

\section{Results and Discussion}

From the methods avaialable for determining large $D$-values (viz. single-crystal EPR spectra, heat capacity measurements, single-crystal magnetic anisotropies, paramagnetic powder susceptibilities, and direct spectroscopic measurements), we have chosen the heat capacity and powder paramagnetic susceptibility at low temperatures, as single crystals of the compounds of sufficiently large size were not available and far-infrared and Raman spectra were expected to be disturbed by interaction with lattice vibrations. 
Figure 1 and 2 show the measured heat capacities in the $1-14 \mathrm{~K}$ region for the chloride and bromide respectively. The high temperature specific heat has been omitted from the figures for clearness. Both salts have an anomalous heat capacity in the He-temperature region. In order to substract the lattice heat capacity a $C T^{2}$ vs. $T^{5}$ plot was made. The estimated lattice heat capacities are drawn in the figures and can be represented by a three-dimensional Debye temperature, $\Theta_{\mathrm{D}}$, of $64.2 \mathrm{~K}$ for the chloride and $63.2 \mathrm{~K}$ for the bromide. The resulting heat capacity after subtraction of the lattice contribution is interpreted as a Schottky anomaly resulting from the $\mathrm{Ni}$ (II) ion, which has a low-lying singlet and a doublet at $7.2 \mathrm{~cm}^{-1}$ for the chloride and at $5.4 \mathrm{~cm}^{-1}$ for the bromide. The best theoretical Schottky heat capacity curves are drawn in the figures.

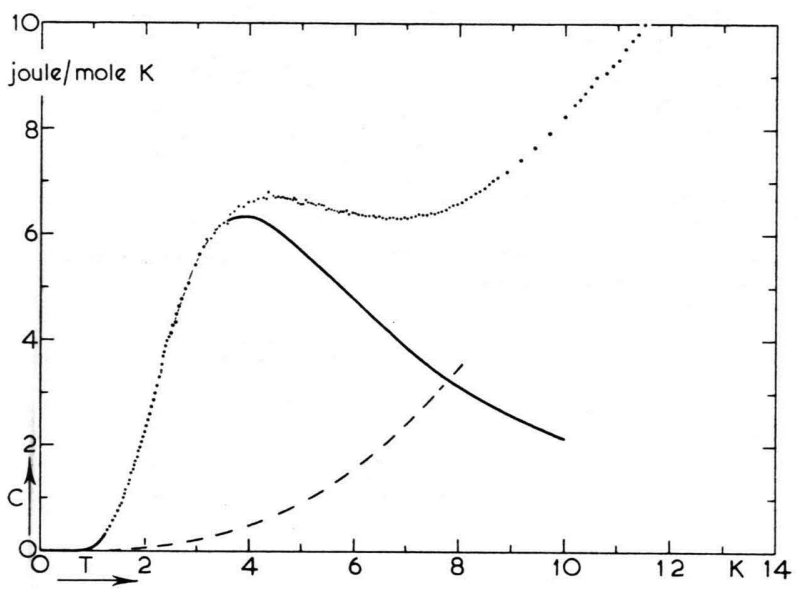

Fig. 1. $C_{p}$ vs. $T$ for $\mathrm{Ni}(\mathrm{Pz}){ }_{4} \mathrm{Cl}_{2}$ (dotted curve). The dashed line is the estimated lattice heat capacity. The full curve is the theoretical Schottky heat capacity anomaly for $D=7.2 \mathrm{~cm}^{-1}$.

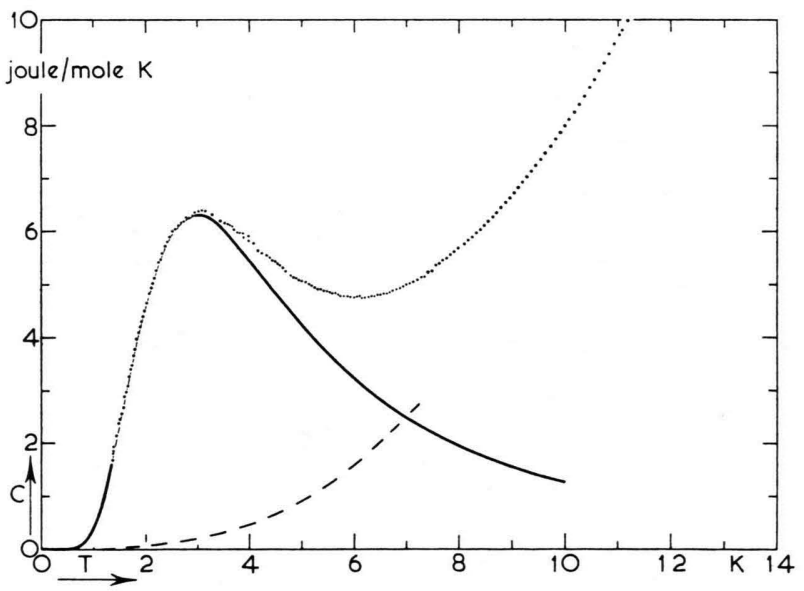

Fig. 2. $C_{p}$ vs. $T$ for $\mathrm{Ni}(\mathrm{Pz})_{4} \mathrm{Br}_{2}$ (dotted curve). The dashed line is the estimated lattice heat capacity. The full curve is the theoretical Schottky heat capacity anomaly for $D=5.4 \mathrm{~cm}^{-1}$
The observed magnetic susceptibility of the compounds was tried fitted with the theoretical expression for $\chi$ as a function of $D, g_{\|}, g_{\perp}$ and $T$. However, the usual theoretical expressions ${ }^{10}, 11$ do not take into account the field dependent part of the susceptibility, and as our compounds were investigated at fields near 5000 Oersted, this would introduce serious errors.

Therefore we made use the basic equation for the susceptibility

$$
\chi=N \Sigma(\partial w / \partial H) \exp \{-w / k T\} / H \Sigma \exp \{-w / k T\}
$$

and calculated the $a b$ initio $\chi$ values directly from this equation and the matrix for the ${ }^{3} \mathrm{~A}_{2} \mathrm{~g}$ ground term under simultaneous zero-field splitting and magnetic field splitting (Table 1).

Table 1. Matrix of simultaneous action on ${ }^{3} \mathrm{~A}_{2} \mathrm{~g}$ by zero-field splittings and magnetic fields.

\begin{tabular}{rccc}
\hline$M_{\mathrm{S}}$ & -1 & 0 & +1 \\
\hline-1 & $D-g_{\|} \beta H_{z}$ & $\sqrt{2} g_{\perp} \beta H_{x} / 2$ & 0 \\
0 & $\sqrt{2} g_{\perp} \beta H_{x} / 2$ & 0 & $\sqrt{2} g_{\perp} \beta H_{x} / 2$ \\
+1 & 0 & $\sqrt{2} g_{\perp} \beta H_{x} / 2$ & $D+g_{\|} \beta H_{z}$ \\
\hline
\end{tabular}

The principal susceptibilities $\chi \|$ and $\chi \perp$ thus obtained, are averaged by the relation

$$
\chi_{\text {powder }}=\frac{2}{3} \chi_{\perp}+\frac{1}{3} \chi_{\|}
$$

and the observed $\chi$ vs. $T$ is compared with several of these theoretical curves. First a rough value for $D$ is obtained from extrapolation to $T \rightarrow 0$ of the expression ${ }^{11}$

$$
\chi_{\text {powder }}(0)=4 N \beta^{2} g_{\perp} 2 / 3 D
$$

with an initial value for $g_{\perp}$ obtained from the averaged value in the powdered compound at high temperature. Then the rough values were refined by fitting with the experimental curves. The splitting in the doublet was found to be very close to zero and is ignored in the fitting.

The results of the best fit of $\chi$ vs. $T$ with $D, g_{\|}, g_{\perp}$ are listed in Table 2, together with the $D$ and $E$ values obtained from the heat capacity measurements. The ex-

\begin{tabular}{|c|c|c|c|c|c|}
\hline \multirow[t]{2}{*}{ Compounds } & \multicolumn{2}{|c|}{ Heat capacity } & \multicolumn{3}{|c|}{ Susceptibility } \\
\hline & $D\left(\mathrm{~cm}^{-1}\right)$ & $E\left(\mathrm{~cm}^{-1}\right)$ & $D\left(\mathrm{~cm}^{-1}\right)$ & $g_{\|}$ & $g_{\perp}$ \\
\hline $\mathrm{Ni}(\mathrm{Pz})_{4} \mathrm{Cl}_{2}$ & $7.2(1) \mathrm{b}$ & $0.0(1)$ & $7.2(2)$ & $2.14(3)$ & $2.21(3)$ \\
\hline $\mathrm{Ni}(\mathrm{Pz})_{4} \mathrm{Br}_{2}$ & $5.4(1)$ & $0.0(2)$ & $5.5(2)$ & $2.12(3)$ & $2.20(3)$ \\
\hline
\end{tabular}

Table 2. Zero-field parameters and $g$-values for compounds $\mathrm{Ni}$ (pyrazole) ${ }_{4} \mathrm{X}_{2}$.

a $E$ stands for the doublet splitting.

b Uncertainties in the last digit are shown between parenthesis. 


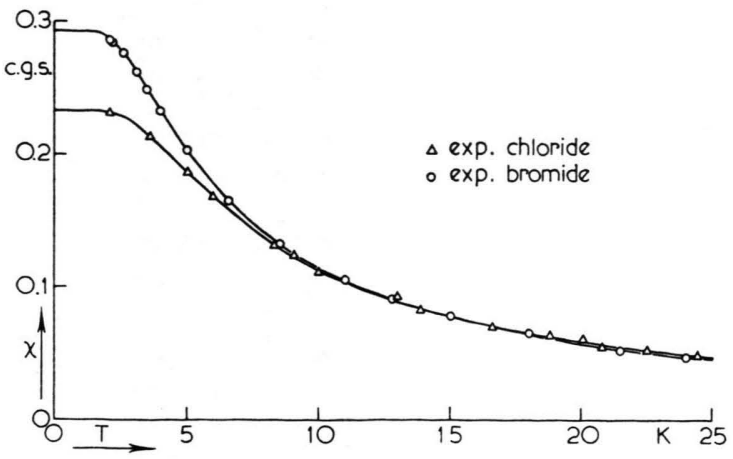

Fig. 3. $\chi$ vs. $T$ for $\mathrm{Ni}(\mathrm{Pz})_{4} \mathrm{X}_{2}$ with $\mathrm{X}=\mathrm{Cl}$ and $\mathrm{Br}$. The calculated curves for the parameters listed in Table 1 are drawn through the experimental points.

1 C. J. Ballhausen, Introduction to Ligand Field Theory, McGraw-Hill, London 1962.

2 J. Reedijk and B. Nieuwenhuijse, Rec. Trav. Chim. 91, 533 [1972].

3 A. D. Liehr and C. J. Ballhausen, Ann. Phys. (New York) 2, 134 [1959].

4 J. ReEdijk, Rec. Trav. Chim. 89, 993 [1970].

5 R. D. Dowsing, B. Nieuwenhuijse, and J. Reedijk, Inorg. Chim. Acta 5, 301 [1971]. perimental $\chi$ vs. $T$ curves are depicted in Figure 3. As is seen from Table 2, the results of both measurements completely overlap, indicating the practical utility of these techniques to obtain zero-field parameters from powder data. The rather large uncertainties in the $g$-values (Table 2) are caused by weighting errors and by slight temperature dependence of the $g$-values.

The magnitude of the $D$-values in the present compounds is quite larger, but smaller and of opposite sign than predicted by LIEHR and BALlHAUSEN ${ }^{3}$ from the orbital splittings ${ }^{4}$.

We are extending these investigations to similar $\mathrm{Ni}$ (II) compounds with other anions and ligands and to calculations of $D$ from the orbital splittings in the excited states, using second-order spin-orbit interactions.

6 J. Reedijk, B. A. Stork-Blaisse, and G. C. Verschoor, Inorg. Chem. 10, 2594 [1971].

7 C. W. Reimann, A. D. Mighell, and F. A. Mauer, Acta Cryst. 23, 135 [1967].

8 A. D. Mighell, C. W. Reimann, and A. Santoro, Acta Cryst. B 25, 595 [1969].

${ }^{9} \mathrm{~F}$. W. KlaAijSEN, to be published.

10 T. Watanabe, J. Phys. Soc. Japan 17, 1865 [1962].

11 R. Prins, J.D. W. van Voorst, and C. J. Schinkel, Chem. Phys. Letters 1, 54 [1967]. 